\title{
Sparse Gaussian Process Emulators for Surrogate Design Modelling
}

\section{Paul Gardner ${ }^{1, \mathrm{a}}$, Timothy J. Rogers ${ }^{1, \mathrm{~b}}$, Charles Lord ${ }^{1, \mathrm{c}}$, and Robert J. Barthorpe ${ }^{1, \mathrm{~d}}$}

\author{
Dynamics Research Group, Department of Mechanical Engineering, University of Sheffield, \\ Sheffield, UK

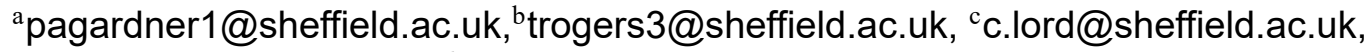 \\ ${ }^{d}$ r.j.barthorpe@sheffield.ac.uk
}

Keywords: Gaussian Process, Sparse Gaussian Process, Kriging, Emulator, Surrogate Modelling.

\begin{abstract}
Efficient surrogate modelling of computer models (herein defined as simulators) becomes of increasing importance as more complex simulators and non-deterministic methods, such as Monte Carlo simulations, are utilised. This is especially true in large multidimensional design spaces. In order for these technologies to be feasible in an early design stage context, the surrogate model (or emulator) must create an accurate prediction of the simulator in the proposed design space. Gaussian Processes (GPs) are a powerful non-parametric Bayesian approach that can be used as emulators. The probabilistic framework means that predictive distributions are inferred, providing an understanding of the uncertainty introduced by replacing the simulator with an emulator, known as code uncertainty. An issue with GPs is that they have a computational complexity of $\mathcal{O}\left(N^{3}\right)$ (where $N$ is the number of data points), which can be reduced to $\mathcal{O}\left(N M^{2}\right)$ by using various sparse approximations, calculated from a subset of inducing points (where $M$ is the number of inducing points). This paper explores the use of sparse Gaussian process emulators as a computationally efficient method for creating surrogate models of structural dynamics simulators. Discussions on the performance of these methods are presented along with comments regarding key applications to the early design stage.
\end{abstract}

\section{Introduction}

Computer models (simulators) are a vital tool in exploring engineering design options. Often design engineers want to investigate numerous different parameter choices in order to find optimal solutions. These design spaces are often large and multidimensional, meaning that evaluation of the simulator at all parameter combinations of interest is infeasible. In order to reduce the computational load of running numerous simulator evaluations at all parameter combinations of interest, an emulator can be used. An emulator is a fast surrogate model of the computationally expensive simulator that allows accurate interpolation of the design space whilst requiring minimal simulator runs. A common approach for constructing an emulator is to fit a Gaussian process (GP) regression model to simulator evaluations at a small number of predefined inputs in order to learn the simulator's input to output mapping [1,2]. Optimisation techniques and uncertainty quantification approaches often involve multiple evaluations of the simulator. An emulator provides significant computational savings in being able to interpolate across the design space with reduced computational complexity. As a result, more complex computer models, optimisation or uncertainty quantification techniques become practicable. An additional strength of this approach is the statistical framework of a GP emulator, which provides an assessment of code uncertainty [3] — uncertainty introduced by approximating the simulator with an emulator. The code uncertainty can also be included within an optimisation setting by using a Bayesian optimisation approach $[4,5]$; or in a calibration setting with methods such as Bayesian history matching [6,7]. The inclusion of code uncertainty means that parts of the input space are not excluded or misrepresented when the emulator is uncertain about the prediction of the simulator.

GPs are a popular method for non-parametric, non-linear, Bayesian regression [8,9]. An issue with GP regression is that training costs $\mathcal{O}\left(N^{3}\right)$ (for $N$ observations) with prediction of the mean 
and variance costing both $\mathcal{O}(N)$ and $\mathcal{O}\left(N^{2}\right)$ respectively [10-12]. Although substantially more computationally efficient than running a simulator, this time complexity can make GPs computationally demanding in circumstances where $N$ is significantly large. To reduce the computational load, sparse approximations of the full GP have been developed over recent years; these have been constructed from a regression standpoint $[10,12-16]$. These sparse approximations reduce the computational complexity of training to $\mathcal{O}\left(N M^{2}\right)$ [10-12] where $M \ll N$ and $M$ is a smaller number of inducing points - a set of pseudo-inputs and their latent function evaluations that are independent from the training data. This paper presents the application of state-of-the-art sparse GP approximations in the context of creating emulators of complex computer models and discusses both model and posterior based approximations and considerations for emulation. The paper outline is as follows: an overview of GP regression is presented, in the following section sparse GP approximations are outlined with discussion on model and posterior approximations as well as implications for emulation. Lastly conclusions are presented highlighting areas of further research.

\section{Gaussian Process Regression}

A simulator can be represented by an underlying functional mapping $f$ between a set of inputs $X$ and their corresponding outputs $Y=f(X)$. It may be possible to run the simulator at any arbitrary set of inputs, however, to evaluate all the combinations of interest is assumed to be computationally expensive; for this reason, only a finite set of $N$ simulator runs are often available. The objective of creating an emulator is to reproduce the functional mapping, via regression, so that predictions of the outputs can be made given new inputs. Even though in this paper it is assumed that the simulator is deterministic, a probabilistic approach is useful as the predictive distributions quantify the uncertainty associated with the prediction, known as code uncertainty [3]. A probabilistic framework means that the mapping between a set of $N$ inputs $X=\left\{\boldsymbol{x}_{n}\right\}_{n=1}^{N}$ of dimension $D$ and their corresponding outputs $\boldsymbol{y}=\left\{y_{n}\right\}_{n=1}^{N}$ is modelled as $p(\boldsymbol{y} \mid \boldsymbol{f}, X, \boldsymbol{\theta})$; where $\boldsymbol{\theta}$ is a small set of hyperparameters. For a GP emulator the latent function $f$ is modelled with a GP prior, and the simulator outputs for different inputs are modelled as jointly Gaussian distributed. The GP prior is formulated as presented in Eq. 1.

$$
p(\boldsymbol{f} \mid X, \boldsymbol{\theta}) \sim \mathcal{N}(\boldsymbol{m}, K)
$$

Where $\boldsymbol{m}$ is a mean function of the form $h^{\prime}(\boldsymbol{x}) \boldsymbol{\beta}$ where $h^{\prime}(\boldsymbol{x})$ is a design matrix and $\boldsymbol{\beta}$ the corresponding hyperparameters. The covariance matrix $K$ defines the prior assumption of the functions smoothness and is formed from the covariance function where $K_{i j}=k\left(x_{i}, x_{j}\right)$, which is dependent on the hyperparameters $\boldsymbol{\theta}_{k}=\left\{\sigma_{f}^{2}, \psi\right\}$ ( $\sigma_{f}^{2}$ is called the signal variance and $\psi$ are some parameters that are dependent on the choice of covariance function). The covariance function describes the correlation between any two points in the input space via a reproducing kernel Hilbert space. An example covariance function is the squared exponential defined in Eqn. 2.

$$
K=\sigma_{f}^{2} \exp \left(\left(-X-X^{\prime}\right) \psi\left(X-X^{\prime}\right)^{T}\right)
$$

A GP depends on a small number of hyperparameters collectively defined as $\boldsymbol{\theta}=\left\{\boldsymbol{\beta}, \boldsymbol{\theta}_{k}\right\}$. The joint prior between the latent function values (for training $f$ and testing $f_{*}$ ) at training and testing inputs, $X$ and $X_{*}$ respectively, can be formed as in Eqn. 3; this uses the definition that a GP is collection of random variables where a finite set has a joint Gaussian distribution ${ }^{1}$.

\footnotetext{
${ }^{1}$ For compactness $\boldsymbol{m}_{a}$ is the mean function relating to the latent function $a$ and $K_{a, b}$ is the covariance matrix between the latent functions $a$ and $b$, e.g. $K_{f, *}$ is the covariance between the training and testing latent functions.
} 


$$
\left[\begin{array}{c}
\boldsymbol{f} \\
\boldsymbol{f}_{*}
\end{array}\right] \sim \mathcal{N}\left(\left[\begin{array}{l}
\boldsymbol{m}_{f} \\
\boldsymbol{m}_{*}
\end{array}\right],\left[\begin{array}{ll}
K_{f, f} & K_{f, *} \\
K_{*, f} & K_{*, *}
\end{array}\right]\right)
$$

The likelihood for the GP emulator is typically modelled as Gaussian, however the variance term of the likelihood is debated. It is common for a GP emulator to assume that the observations are "noisefree', i.e. repeats at the same set of inputs will always result in the same output — for a deterministic simulator. Even so, due to numerical instabilities in inverting the covariance matrix, GP regression becomes impractical unless a nugget term is added; usually a fixed small number to the diagonal of the covariance matrix. This can be seen as equivalent to assuming a small level of Gaussian white noise on the observation, as assumed in many other fields such as kriging and general regression $[9,17]$ where the likelihood is formed as $p(\boldsymbol{y} \mid \boldsymbol{f})=\mathcal{N}\left(\boldsymbol{f}, \sigma_{n}^{2} I\right)$; where $\sigma_{n}^{2}$ is the variance associated with the noise. The inclusion of the nugget is known to sometimes lead to inference of the latent function $f$ that smooths through data points with a variance rather than fitting known input, output pairs exactly. Andrianakis and Challenor proposed a penalty to the marginal likelihood in order to force a GP emulator with a nugget term to fitting known data points exactly [18]. In this paper a nugget term will be included and assumed to take a very small value. The resulting likelihood is of the form $p(\boldsymbol{y} \mid \boldsymbol{f})=\mathcal{N}(\boldsymbol{f}, \nu I)$; where $\nu$ is the nugget term.

In order to perform inference the joint prior is combined with the likelihood to form the joint posterior $p\left(\boldsymbol{f}, \boldsymbol{f}_{*} \mid \boldsymbol{y}, \boldsymbol{\theta}\right)$ using Bayes rule (the inputs are dropped for simplicity of notation). The latent training function $f$ can then be marginalised out form the posterior shown in Eqn. 4; this is possible in closed form due to the Gaussian assumptions.

$$
p\left(\boldsymbol{f}_{*} \mid \boldsymbol{y}, \boldsymbol{\theta}\right)=\mathcal{N}\left(\boldsymbol{m}_{*}+K_{*, f}\left(K_{f, f}+\nu I\right)^{-1}(\boldsymbol{y}-\boldsymbol{m}), K_{*, *}-K_{*, f}\left(K_{f, f}+\nu I\right)^{-1} K_{f, *}\right)
$$

A fully Bayesian analysis would then require marginalising out the hyperparameters $\theta$, this is possible for $\boldsymbol{\beta}$ and the signal variance $\sigma_{f}^{2}$ [19], but is intractable for the parameters $\psi$. If an MCMC solution to the hyperparameters is used then this marginalisation provides valuable computational savings. On the other hand if a maximum likelihood approach is used, the computational savings of the marginalisation are minimal as this becomes the optimisation of a small number of parameters. In this paper, a maximum likelihood approach is used due to it being a fast and efficient form of inference with the only negative being that the posterior variance of the GP is slightly underestimated. Inference of the hyperparameters using a maximum likelihood approach requires forming the marginal likelihood, the integral of the prior multiplied by the likelihood, the log form of which is shown in Eqn. 5.

$$
\log p(\boldsymbol{y} \mid X, \boldsymbol{\theta})=-\frac{1}{2} \log \left|K_{f, f}+\nu I\right|-\frac{1}{2}\left(\boldsymbol{y}-\boldsymbol{m}_{f}\right)^{T}\left(K_{f, f}+\nu I\right)^{-1}\left(\boldsymbol{y}-\boldsymbol{m}_{f}\right)-\frac{N}{2} \log 2 \pi
$$

The problem with Eqn. 4 and Eqn. 5 is their dependence on inverting an $N \times N$ matrix requiring $\mathcal{O}\left(N^{3}\right)$ operations [10-12]. If a computationally efficient algorithm is used, the prediction complexity of Eqn. 4 is only $\mathcal{O}(N)$ with the variance being $\mathcal{O}\left(N^{2}\right)$. In order to improve this computational complexity sparse approximations are implemented. This paper will assume a zero mean function in order to simplify notation, however, it is trivial to include a mean function. In keeping with the brevity of this paper the reader is referred to $[8,9,19]$ for more mathematical definitions and explanations of GPs. 


\section{Sparse GP Approximations}

Sparse GP approximations seek to reduce the computational load involved in inverting $K_{f, f}$. The simplest and most naive approach is to select a subset of data (SoD) of size $Q$ from the full training data set (of size $N$ where $Q \ll N$ ) in order to scale down the time complexity to $\mathcal{O}\left(Q^{3}\right)$ [10]. The problem is difficult as it relies on a known redundancy within the original data set, which is often not the case - especially in expensive evaluations of a simulator. This loss of information is generally unacceptable in an emulation context, as any simulator runs are expected to have come at a large computational cost. An alternative to SoD is the local GP approach [11]. A simple implementation of local GPs is to divide a data set into equal block sizes of size $B$ and fit a GP to each block; reducing the computational complexity to $\mathcal{O}\left(N B^{2}\right)$. An issue with the technique is that discontinuities will occur between each data block, this is often unacceptable in emulators due to their primary use in optimisation or calibration. A less naive implementation of the local GP approach is to use a clustering algorithm to categorise the data into various subsets and fit GP models to each subset of data. As a consequence, the computational complexity of the method will not only be dominated by the largest subset, but will also incur the additional cost of the clustering algorithm. Both SoD and local GP approaches are therefore not ideal for an emulator setting.

This paper explores two key ideas in the generation of sparse GP approximations; approximating the model or the posterior. The techniques use inducing inputs [10] (originally referred to as 'pseudoinputs' [13]) $Z=\left\{\boldsymbol{z}_{m}\right\}_{n=1}^{M}$, that have a latent function output $\boldsymbol{u}$, known as inducing variables in order to produce sparsity.

Model Approximation Approaches. Quiñonero-Candela and Rasmussen present a unified framework for model approximations [10]. These approaches seek to modify the joint prior $p\left(\boldsymbol{f}_{*}, \boldsymbol{f}\right)$ of the GP (Eqn. 3) in order to replace the complexity of inverting $K_{f, f}$ with a less expensive inversion. This is performed by incorporating inducing points $\{Z, \boldsymbol{u}\}$ (where $Z$ are a set of inducing inputs and $\boldsymbol{u}$ are the corresponding latent function evaluations) into the joint prior $p\left(\boldsymbol{f}_{*}, \boldsymbol{f}, \boldsymbol{u}\right)$ and marginalising the inducing variables, $\boldsymbol{u}$, out of the posterior (although $Z$ will affect the final solution). The key assumption for these sparse methods is that the joint prior can be approximated by assuming conditional independence between $\boldsymbol{f}_{*}$ and $\boldsymbol{f}$ given $\boldsymbol{u}$. This means that $\boldsymbol{f}_{*}$ and $\boldsymbol{f}$ are only linked through $\boldsymbol{u}$; demonstrated in Eqn. 6.

$$
p\left(\boldsymbol{f}_{*}, \boldsymbol{f}\right) \simeq q\left(\boldsymbol{f}_{*}, \boldsymbol{f}\right)=\int p\left(\boldsymbol{f}_{*} \mid \boldsymbol{u}\right) q(\boldsymbol{f} \mid \boldsymbol{u}) p(\boldsymbol{u}) d \boldsymbol{u}
$$

Where $p(\boldsymbol{u})=\mathcal{N}\left(0, K_{u, u}\right)$ is the prior ${ }^{2}$ for the latent variables $\boldsymbol{u}$ and the test conditional, $p\left(\boldsymbol{f}_{*} \mid \boldsymbol{u}\right)$, is defined in Eqn. 7.

$$
p\left(\boldsymbol{f}_{*} \mid \boldsymbol{u}\right)=\mathcal{N}\left(K_{*, u} K_{u, u}^{-1} \boldsymbol{u}, K_{*, *}-Q_{*, *}\right)
$$

It is noted that the notation $Q_{a, b}=K_{a, u} K_{u, u}^{-1} K_{u, b}$ is used in this paper. The two model approximation methods detailed in this paper differ in their assumption about the training conditional $q(\boldsymbol{f} \mid \boldsymbol{u})$, whilst assuming the same prior for the inducing variables and likelihood.

The assumptions for the training conditional $q(\boldsymbol{f} \mid \boldsymbol{u})$, and the marginalised joint prior $p\left(\boldsymbol{f}_{*}, \boldsymbol{f}\right)$, for both a deterministic training conditional (DTC) and fully independent training conditional (FITC) approximation are shown in Table 1. The main difference between DTC and FITC is clear in the joint prior, presented in Table 1. The top left corner of the covariance is modified in FITC so that the

\footnotetext{
${ }^{2}$ It is common for a nugget, $\epsilon I$ to be incorporated here [20] for the same reasons as outline for emulators previously, i.e. increases the stability of the inversion of the covariance matrix. A nugget is used in this paper meaning $p(\boldsymbol{u})=\mathcal{N}\left(0, K_{u, u}+\epsilon I\right)$.
} 
approximation includes the exact covariance on the diagonal. This transforms the training conditional from deterministic to fully independent.

The posteriors $q\left(\boldsymbol{f}_{*} \mid \boldsymbol{y}, \boldsymbol{\theta}\right)$ and log marginal likelihoods $p(\boldsymbol{y} \mid X)$ for the DTC and FITC approximations can be unified into the analytical form outlined in Eqn. 8 and Eqn. 9. [12].

$$
q\left(\boldsymbol{f}_{*} \mid \boldsymbol{y}, \boldsymbol{\theta}\right)=\mathcal{N}\left(Q_{*, f} \bar{K}_{f, f}^{-1} \boldsymbol{y}, K_{*, *}-Q_{*, f} \bar{K}_{f, f}^{-1} Q_{f, *}\right)
$$

Table 1: DTC and FITC assumptions for the training conditional $q(\boldsymbol{f} \mid \boldsymbol{u})$ and the joint prior $p\left(\boldsymbol{f}_{*}, \boldsymbol{f}\right)$ (where $\operatorname{diag}[A]$ refers to the diagonal of matrix $A$ ). The joint prior $p\left(\boldsymbol{f}, \boldsymbol{f}_{*}\right)$ is calculated by substituting the training condition $q(\boldsymbol{f} \mid \boldsymbol{u})$ into Eqn. 6 and solving the integral which can be done in closed form.

\begin{tabular}{lcc}
\hline Method & Training Conditional, $q(\boldsymbol{f} \mid \boldsymbol{u})$ & Joint Prior, $p\left(\boldsymbol{f}_{*}, \boldsymbol{f}\right)$ \\
\hline DTC & $\mathcal{N}\left(K_{f, u} K_{u, u}^{-1} \boldsymbol{u}, 0\right)$ & $\mathcal{N}\left(\mathbf{0},\left[\begin{array}{cc}Q_{f, f} & Q_{f, *} \\
Q_{*, f} & K_{*, *}\end{array}\right]\right)$ \\
& $\mathcal{N}\left(K_{f, u} K_{u, u}^{-1} \boldsymbol{u}, \operatorname{diag}\left[K_{f, f}-Q_{f, f}\right]\right)$ & $\mathcal{N}\left(\mathbf{0},\left[\begin{array}{cc}Q_{f, f}-\operatorname{diag}\left[Q_{f, f}-K_{f, f}\right] & Q_{f, *} \\
Q_{*, f} & K_{*, *}\end{array}\right]\right)$ \\
\hline
\end{tabular}

$$
\log p(\boldsymbol{y} \mid X)=-\frac{1}{2} \log \left|\bar{K}_{f, f}\right|-\frac{1}{2} \boldsymbol{y}^{T} \bar{K}_{f, f}^{-1} \boldsymbol{y}-\frac{N}{2} \log 2 \pi
$$

Where $\bar{K}_{f, f}=Q_{f, f}+\operatorname{diag}\left[\alpha\left(K_{f, f}-Q_{f, f}\right)\right]+\nu I$. The marginal likelihood and posterior of the two methods can be formulated by setting $\alpha$ to zero or one for the DTC and FITC approximations

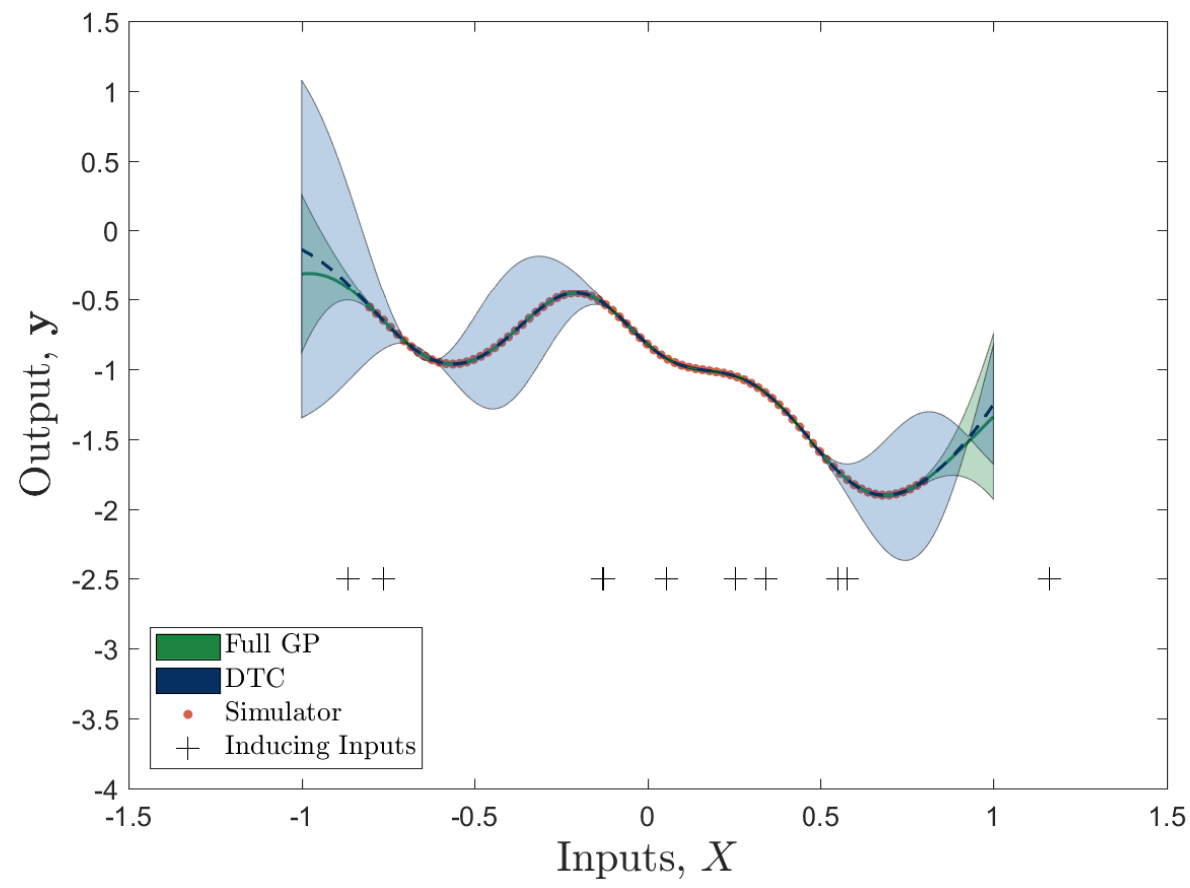

Fig. 1: Predictions from a sparse DTC GP with 10 inducing points, against a full GP and training simulator data for a numerical example. Shaded regions indicate $\pm 3 \sigma$ confidence levels. 
respectively. After setting $\alpha$, the low rank structure of $\bar{K}_{f, f}$ should be exploited using the Woodbury inversion and determinant lemmas in order to improve the computational efficiency. These amendments reduce the computational complexity for training to $\mathcal{O}\left(N M^{2}\right)$ and for prediction to $\mathcal{O}(M)$ and $\mathcal{O}\left(M^{2}\right)$ for the mean and variance respectively [10-12].

The question remains of how to set the inducing inputs. One approach is to consider the inducing inputs as a subset of the input data. This poses challenges when global prediction quality is required as the selection of inducing inputs from a discrete set of data will involve some form of greedy or combinatorial optimisation. In contrast, the inducing inputs can be considered to be drawn from any point on the real line leading to a continuous optimisation problem [13]. This allows the inducing inputs to be inferred via optimisation of the log marginal likelihood. When the inducing inputs are equal to the training inputs, the marginal likelihood and the posterior are the same as the full GP for both DTC and FITC. A key drawback of model approximation methods are that optimising via the approximate marginal likelihood means treating the inducing inputs as parameters of the model, adding all the problems of overfitting and optimisation that are evident in parametric models [12,14]. This view of the inducing points means the assumptions about the data and inference approximations are coupled. Learning via the exact marginal likelihood of the approximate model also means that the hyperparameters will be optimal for the approximate model and not necessarily the full GP.

Figure 1 and Fig. 2 present univariate numerical examples where the simulator output is a sample from GP process (a random seed integer of 5 is used in MATLAB) with zero mean and a squared exponential covariance; $\sigma_{f}^{2}=1$ and $\psi=8$. The examples demonstrate the difference in the two approaches when the hyperparameters $\theta$ and inducing inputs $Z$ are learnt through optimising the $\log$ marginal likelihood $\log p(\boldsymbol{y} \mid X)$ (Eqn. 9). These illustrate a comparison of the two sparse GP methods, DTC and FITC, with a full GP solution and the training data, where the mean and $\pm 3 \sigma$ confidence intervals are displayed for the full and sparse GPs (indicated by the shaded regions). It is shown that FITC gives a better approximation of the variance than DTC, that tends to overestimate (due to the deterministic assumption). Signs of overfitting are present in both methods. In Fig. 1 the variance for DTC when $X \approx 0.9$ reduces almost to zero, displaying overconfidence in the prediction when it would be expected to increase from the last training point, as shown in the full GP solution. The FITC approach in Fig. 2 visually fits the middle section of training data well, however the variance starts to increase before the ends of the training data. This indicates that the inducing points have been placed in locations that overfit the middle section of the training data, leading to poor generalisation at the edges of the training data set.

Posterior Approximation Approaches. An alternative approach to model approximations is to apply sparsity at the inference stage; this means approximating the posterior and marginal likelihood. Here two approaches are consider; variation free energy (VFE) [14] and power expectation propagation (PEP), where PEP has been shown to be a framework unifying both VFE and FITC [12].

VFE aims to approximate the true posterior by constructing a variational approximation maximising the evidence lower bound (which is a lower bound of the $\log$ marginal likelihood $\log p(\boldsymbol{y} \mid X)$ ). VFE is a specific form of variational inference which can also be formulated in a more general sense (with a uncollapsed form of the bound) [15]. VFE incorporates the inducing inputs as parameters of the variational inference removing problems associated with treating them as model parameters. A variational posterior is proposed, augmented by the inducing variables, where $q(\boldsymbol{f}, \boldsymbol{u})=p(\boldsymbol{f}, \boldsymbol{u}) \phi(\boldsymbol{u})$ and $\phi(\boldsymbol{u})$ is defined as a 'free' Gaussian distribution with $\boldsymbol{u}$ depending on the 'free' inputs $Z$. The inducing inputs $Z$ and the 'free' distribution $\phi(\boldsymbol{u})$ can be specified by minimising the distance between the variational distribution and the augmented true posterior distribution $p(\boldsymbol{f}, \boldsymbol{u} \mid \boldsymbol{y})$, using the KullbackLeibler (KL) divergence, $K L(q(\boldsymbol{f}, \boldsymbol{u}) \| p(\boldsymbol{f}, \boldsymbol{u} \mid \boldsymbol{y}))$. As stated, this is equivalent to maximising the lower bound of the true log marginal likelihood as defined as in Eqn. 10. 


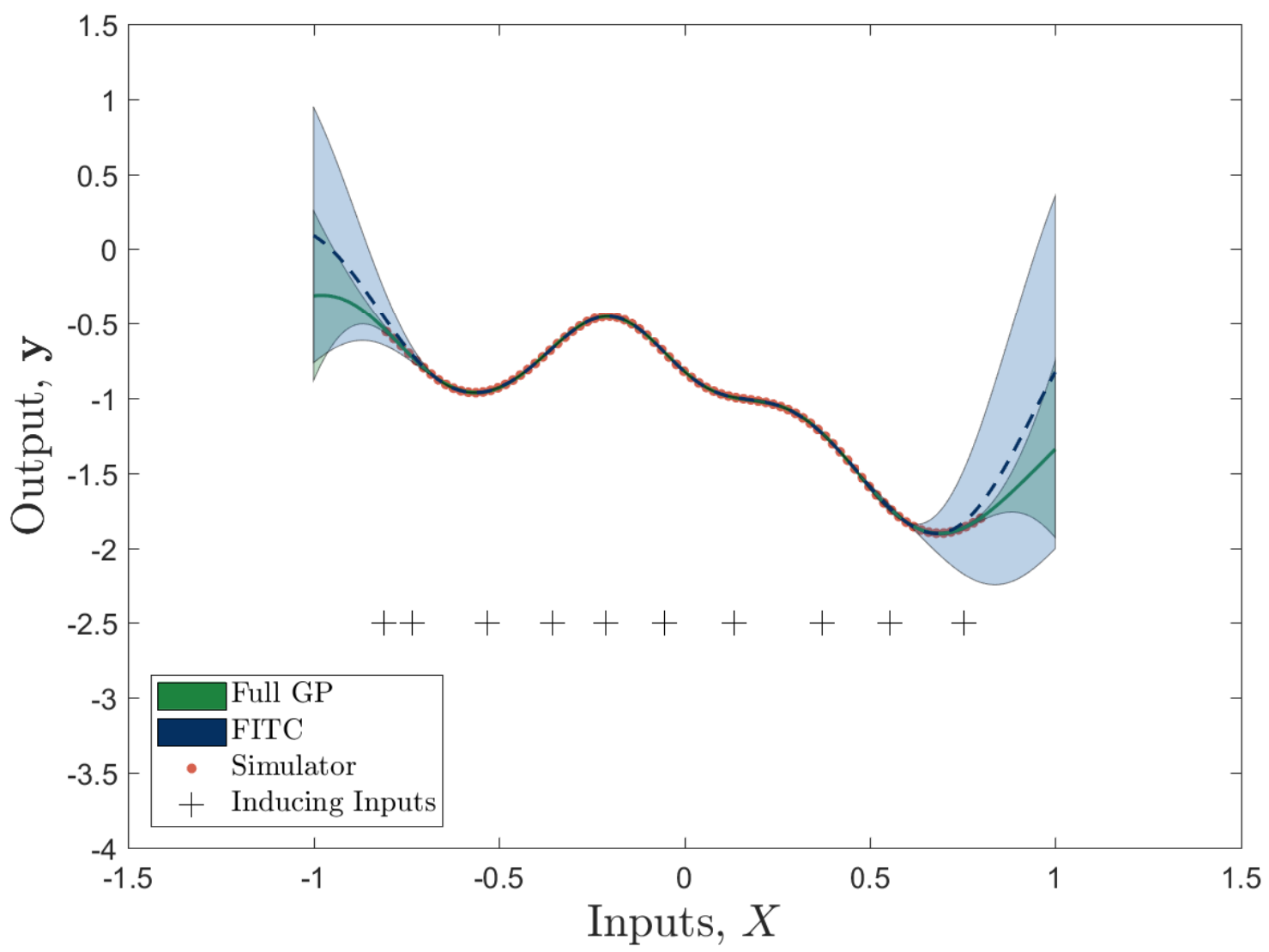

Fig. 2: Predictions from a sparse FITC GP with 10 inducing points, against a full GP and training simulator data for a numerical example. Shaded regions indicate $\pm 3 \sigma$ confidence levels.

$$
F_{v}(Z, \phi)=\int p(\boldsymbol{f} \mid \boldsymbol{u}) \phi(\boldsymbol{u}) \log \frac{p(\boldsymbol{y} \mid \boldsymbol{f}) p(\boldsymbol{f}+\boldsymbol{u}) p(\boldsymbol{u})}{\underline{p(\boldsymbol{f}+\boldsymbol{u}) \phi(\boldsymbol{u})}} d \boldsymbol{f} d \boldsymbol{u}
$$

The optimal choice for the 'free' distribution $\phi(\boldsymbol{u})$ can be found analytically by using variational calculus resulting in the Eqn. 11 [14].

$$
F_{v}(Z)=-\frac{1}{2} \log \left|Q_{f, f}+\nu I\right|-\frac{1}{2} \boldsymbol{y}^{T}\left(Q_{f, f}+\nu I\right)^{-1} \boldsymbol{y}-\frac{N}{2} \log 2 \pi-\frac{1}{2 \nu} \operatorname{Trace}\left(K_{f, f}-Q_{f, f}\right)
$$

Equation 11 is equivalent to that of DTC with the inclusion of a trace regularisation term. This means that the objective function in the optimisation is a true lower bound of the marginal likelihood. Following the analysis through with the optimal 'free' distribution the approximate posterior is formed as defined in Eqn. 12.

$$
q\left(\boldsymbol{f}_{*} \mid \boldsymbol{y}, \boldsymbol{\theta}\right)=\mathcal{N}\left(Q_{*, f} \tilde{K}_{f, f}^{-1} \boldsymbol{y}, K_{*, *}-Q_{*, f} \tilde{K}_{f, f}^{-1} Q_{f, *}\right)
$$

Where $\tilde{K}_{f, f}=Q_{f, f}+\nu I$. The approximate posterior is identical to that of DTC and therefore VFE can be thought of as DTC but penalised by a term proportional to the summed variances. This term protects against overfitting and forces the inducing inputs to better explain all the data, improving their optimised locations. The approach remains non-parametric and because the inducing points become variational parameters any additional inducing points will always increase the prediction quality, which cannot be claimed for model approximation methods. 
An additional approach to approximating the posterior is to use a PEP framework [12]. The method seeks to approximate the joint-distribution in the form of Eqn. 13.

$$
p\left(\boldsymbol{f}_{*}, \boldsymbol{y} \mid \boldsymbol{\theta}\right)=p\left(\boldsymbol{f}_{*} \mid \boldsymbol{y}, \boldsymbol{\theta}\right) p(\boldsymbol{y} \mid \boldsymbol{\theta}) \approx p\left(\boldsymbol{f}_{*} \mid \boldsymbol{\theta}\right) \prod_{n} t_{n}(\boldsymbol{u})=q^{u n}\left(\boldsymbol{f}_{*} \mid \boldsymbol{\theta}\right)
$$

Where $(\cdot)^{u n}$ indicates an unnormalised process. Eqn. 13 shows that only the likelihood term in the exact posterior is approximated and by a factor $t_{n}(\boldsymbol{u})$ assumed to be Gaussian. PEP then iteratively modifies the factors in order to capture the behaviour the true likelihood imposes on the posterior, i.e. the best surrogate likelihood that approximates the posterior. The PEP algorithm involves three steps in which a fraction $\alpha$ of the approximate likelihood function is incorporated iteratively for each factor that needs to be approximated.

1. Deletion: a fraction of one approximate factor is removed in order to evaluated the cavity distribution (this is an approximate leave-one out joint, where $\backslash_{n}$ indicates leave-one out $)^{3}$, $q_{\backslash n}^{u n}\left(\boldsymbol{f}_{*}, \boldsymbol{\theta}\right) \propto q^{u n}\left(\boldsymbol{f}_{*}, \boldsymbol{\theta}\right) / t_{n}^{\alpha}(\boldsymbol{u})$.

2. Projection: a tilted distribution is projected onto the posterior distribution using the alpha-divergence for unnormalised densities. $q_{\backslash n}^{u n}\left(\boldsymbol{f}_{*}, \boldsymbol{\theta}\right) \leftarrow \arg \min \bar{D}_{\alpha}\left(\tilde{p}\left(\boldsymbol{f}_{*}\right) \| q_{{ }_{n}}^{u n}\left(\boldsymbol{f}_{*}, \boldsymbol{\theta}\right)\right)$. The titled distribution is formulated by using the same fraction of the true likelihood as used in creating the cavity distribution, $\tilde{p}\left(\boldsymbol{f}_{*}\right)=q_{\backslash n}^{u n}\left(\boldsymbol{f}_{*}, \boldsymbol{\theta}\right) p^{\alpha}\left(y_{n} \mid f_{n}\right)$.

3. Update: An updated factor is calculated by the inclusion of a new fraction of the approximate factor, $t_{n}(\boldsymbol{u})=t_{n, \text { old }}^{1-\alpha}(\boldsymbol{u}) t_{n, \text { new }}^{\alpha}(\boldsymbol{u})$ where $t_{n, \text { new }}^{\alpha}(\boldsymbol{u})=q^{u n}\left(\boldsymbol{f}_{*}, \boldsymbol{\theta}\right) / q_{\backslash n}^{u n}\left(\boldsymbol{f}_{*}, \boldsymbol{\theta}\right)$.

As the GP model defined in this paper has a Gaussian likelihood, the PEP approach has a closed form solution. This is because the approximate factors can be defined at convergence as stable fixed points and the update step remains the same. This results in the approximate log marginal likelihood $\log \mathcal{Z}_{P E P}$ and posterior $q\left(\boldsymbol{f}_{*} \mid \boldsymbol{y}, \boldsymbol{\theta}\right)$ defined in Eqn. 14 and Eqn. 8; where the posterior is equivalent to the approximate model approach (for brevity of this paper see Bui et al. [12] for complete derivations).

$$
\log \mathcal{Z}_{P E P}=-\frac{1}{2} \log \left|\bar{K}_{f, f}\right|-\frac{1}{2} \boldsymbol{y}^{T} \bar{K}_{f, f}^{-1} \boldsymbol{y}-\frac{N}{2} \log 2 \pi-\frac{1-\alpha}{2 \alpha} \sum_{n} \log \left(1+\alpha D_{f_{n}, f_{n}} / \nu I\right)
$$

Where $D_{f, f}=K_{f, f}-Q_{f, f}$. Interesting results occur when $\alpha=1$ and as $\alpha \rightarrow 0$, the PEP posterior and log marginal likelihood become equivalent to the FITC and VFE approach respectively. This unifying view is helpful in understanding the effects of the parameter $\alpha$. When $\alpha<1$ the last term of the PEP $\log$ marginal likelihood $\frac{1-\alpha}{2 \alpha} \sum_{n} \log \left(1+\alpha D_{f_{n}, f_{n}} / \nu I\right)$ will act as a regularising term and making sure that the model generalises well to new outputs; the extreme of the penalty term being the VFE trace term. Bauer et al. produced an overview of the differences between the FITC and VFE approaches [20]. They state that FITC has several negative drawbacks, it can overestimate the marginal likelihood, underestimate the noise/nugget, is not guaranteed to improve when more inducing points are added and does not recover the true posterior. VFE in contrast, can overestimate the noise/nugget, does improve with more inducing points and will recover the true posterior where possible whilst providing a true lower bound of the marginal likelihood. When employing a posterior approximation approach, the nugget term will need to be inferred as a hyperparameter, rather than a fixed term. This is because the nugget now includes a measure of the uncertainty introduced by using a low rank approximation when performing inference. It is noted that both VFE and PEP approximations results in a computational complexity of $\mathcal{O}\left(N M^{2}\right)$ for training with $\mathcal{O}(M)$ and $\mathcal{O}\left(M^{2}\right)$ for the mean and variance predictions $[12,14]$.

$$
{ }^{3} p_{\backslash n}^{*}\left(\boldsymbol{f}_{*}, \boldsymbol{\theta}\right)=p\left(\boldsymbol{f}_{*}, \boldsymbol{y} \mid \boldsymbol{\theta}\right) / p\left(y_{n} \mid f_{n}, \boldsymbol{\theta}\right) \approx q_{\backslash n}^{u n}\left(\boldsymbol{f}_{*}, \boldsymbol{\theta}\right)=q^{u n}\left(\boldsymbol{f}_{*}\right) / t_{n}(\boldsymbol{u})
$$



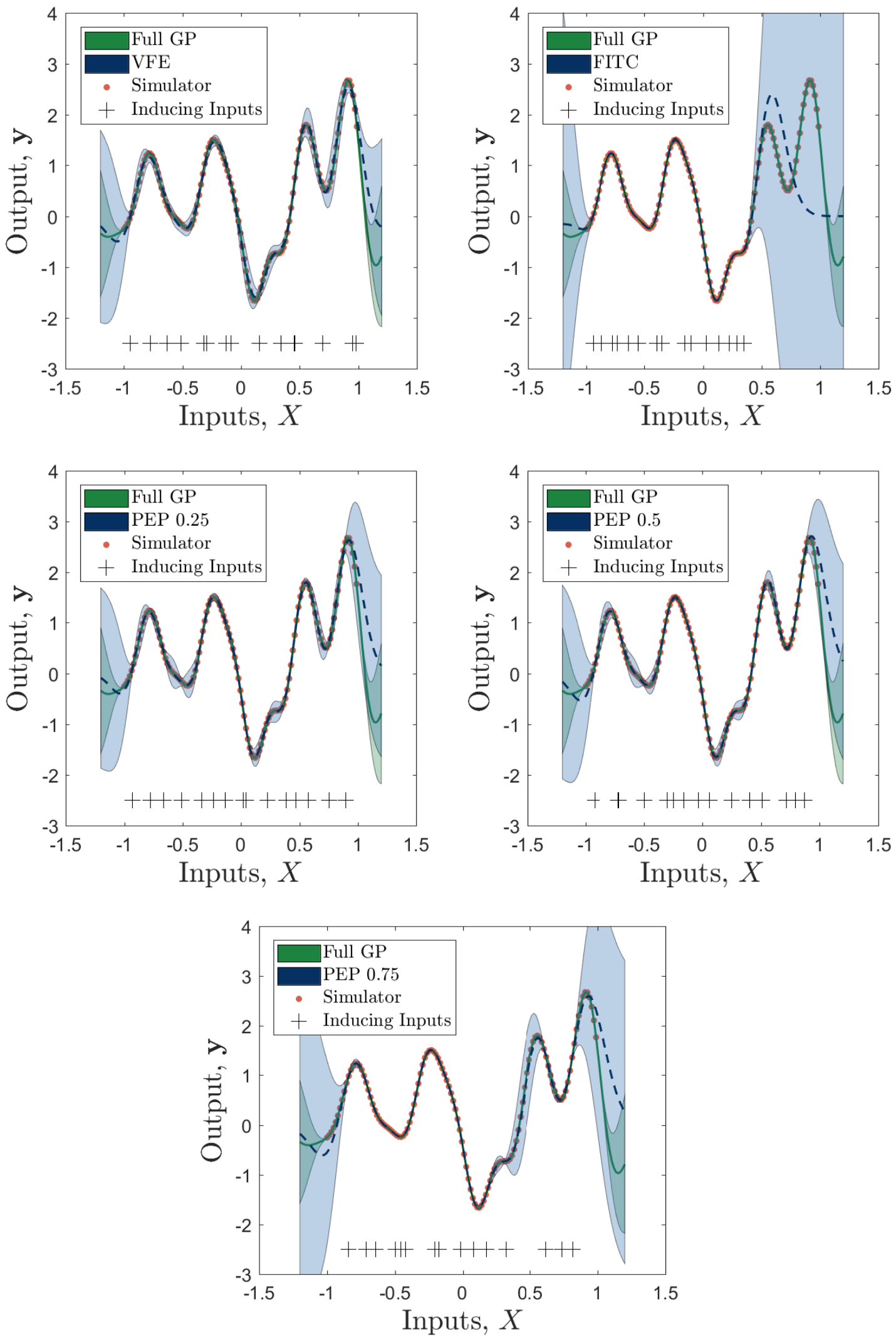

Fig. 3: Predictions from posterior approximation and FITC sparse GPs with 15 inducing points, against a full GP and training simulator data for a numerical example. Shaded regions indicate $\pm 3 \sigma$ confidence levels. Top left panel, VFE $(\alpha=0)$; top right panel, FITC $(\alpha=1)$; middle left panel, PEP $\alpha=0.25$; middle right panel, PEP $\alpha=0.5$; and bottom panel PEP $\alpha=0.75$. 

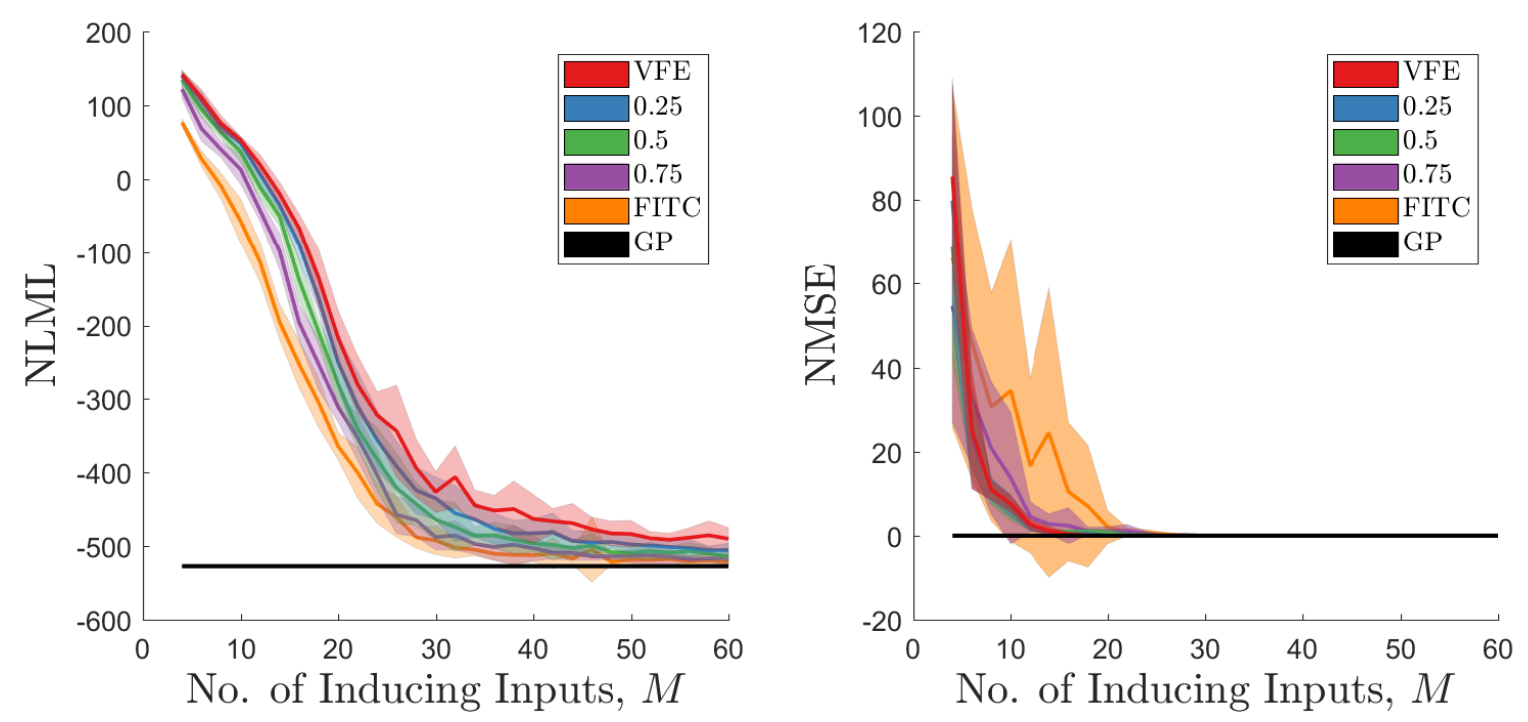

Fig. 4: The effect of the number of inducing inputs $M$ and $\alpha$ on the performance of the PEP formulation of sparse GPs averaged over 25 repeats for the negative log marginal likelihood (NLML, left) and the normalised mean squared error (NMSE, right). Shaded regions indicate $\pm \sigma$ confidence intervals.

Figure 3 and Fig. 4 demonstrate the effect of additional inducing points and the $\alpha$ parameter for a different one-dimensional numerical example. Here the simulator output is a sample from a GP (random seed integer of 5 is used in MATLAB) with zero mean and a squared exponential covariance function; $\sigma_{f}^{2}=1, \psi=30$. Sparse GPs models were created with $\alpha=0,0.25,0.5,0.75,1$ and are compared to the full GP solution and training data in Fig. 3, where mean and $\pm 3 \sigma$ confidence intervals are presented. A stochastic optimisation method was utilised for inferring the hyperparameters with 25 repeats to quantify the variance in the inference, presented in Fig. 4 as $\pm \sigma$ confidence intervals. It is demonstrated that the negative log marginal likelihoods (NLML) $(-\log p(\boldsymbol{y} \mid X)) \approx-F_{v}(Z) \approx$ $\left.-\log \mathcal{Z}_{P E P}\right)$ reduce as more inducing points are added, stating that the model better explains the data given more inducing inputs. The NLML also increases with $\alpha$, in contrast, the normalised mean squared error (NMSE) (Eqn. 15) is high with larger variance for FITC, a clear indication that the method has experienced overfitting. It is noted that there is significant overlap in NMSE results for VFE and PEP when $\alpha=0.25,0.5$, indicating their predictions are very similar.

$$
N M S E=\frac{100}{N \sigma_{y}^{2}} \sum\left(\boldsymbol{f}_{*}-\boldsymbol{y}\right)^{2}
$$

Where $\boldsymbol{f}_{*}$ is the mean prediction of the GP emulator, $\boldsymbol{y}$ and $\sigma_{y}^{2}$ are a set of true simulator outputs and their variance respectively. The NMSE formulation means that a score of zero indicates a mean prediction without error and a score of 100 for scenarios where the prediction is no better than taking the mean of the true values.

The PEP approach, when $\alpha=0.25,0.5$ provides better predictions of the data when compared to FITC and PEP at $\alpha=0.75$, demonstrated by low NLMLs that correspond to low NMSEs. FITC and PEP when $\alpha=0.75$, although showing low NLMLs, have high NMSEs with large variance, especially when the number of inducing points is low; which is a clear sign of overfitting. VFE tends to have high NLML with comparable NMSE to PEP when $\alpha=0.25,0.5$. Figure 3 demonstrates that the variance of the VFE prediction is larger than the full GP solution, with the variance of both the PEP formulations when $\alpha=0.25,0.5$ visually matching the full GP more closely. For these reasons it can be argued that PEP when $\alpha=0.25,0.5$ preforms better in these examples. A close inspection of the NMSE in Fig. 4 for PEP when $\alpha=0.5$, demonstrates lower values than any of the posterior approximation methods. This leads to the conclusion that PEP with an $\alpha=0.5$ outperforms other $\alpha$ 
values (FITC and VFE included) which is consistent with the findings of Bui et al [12]. The question still arises of how to choose the $\alpha$ parameter. Optimisation is not advised as a value of 1 will lead to overfitting due to the FITC approximation. It is the experience of the authors that a value of 0.5 should give satisfactory performance, in-keeping with the finding of Bui et al. [12].

Considerations for Sparse GP Emulators. There are two main reasons why a sparse GP approximation can be useful in creating an emulator. Firstly, when a relatively large number of simulator runs are available, a sparse approximation can make inference practical. This is achieved by reducing the computational time complexity to $\mathcal{O}\left(N M^{2}\right)$ per simulator observation and reducing the memory requirement. Secondly, when predictions are required at a large number of test inputs a moderate computational saving is made, $\mathcal{O}(M)$ and $\mathcal{O}\left(M^{2}\right)$ per test point. Applications of when these reasons may be applicable is presented in this section.

In the authors opinion, it is not commonplace that the simulator is run at a large number of parameter combinations. This problem mainly arises in a high dimensional parameter space where most of the parameters actively and significantly effect the output. Here even a space-filled design will result in a large number of simulator runs and a sparse GP approximation is applicable. Sparse GPs are more useful in a Bayesian optimisation $[4,5]$ or Bayesian history matching setting $[6,7]$. Both methods often require predictions from the emulator for a large number of parameter combinations in order to accurately assess the output space for optimal solutions. The moderate computational saving in the prediction per test point means that a better exploration of the space can be performed. This becomes more important in a sequential design process for adding additional simulator runs to the optimisation, as used in an entropy search or information gain approach [4]. These methods often predict based on a set grid size for the parameter space; reducing the computational load for prediction means a finer grid can be set. Due to the approximate nature of sparse GPs their use is not always needed or favourable for creating emulators. The approximation introduces a nugget term that cannot be fixed as it is a coupling between a noise parameter for the data and an estimation of the error introduced by a low rank approximation. This means that deterministic predictions at know simulator outputs are not possible, as is the case with the full GP emulator. This has to be considered when the code uncertainty affects the results of additional processes, as is the case with Bayesian optimisation and Bayesian history matching.

\section{Duffing Oscillator Case Study}

A case study is presented showing emulation of a design parameter space for a duffing oscillator defined in Eqn. 16 - as motivation for the use of sparse GPs as emulators within an early design context. The objective of this case study is to demonstrate the ability of a sparse GP, using the PEP formulation with $\alpha=0.5$ (due to the reasoning from the previous section), to emulator a large design space based. Specificity, the aim is to emulate a $5 \times 5$ parameter grid of stiffness $k$ and cubic stiffness $k_{3}$ terms that relate to a 512 point displacement time history $\boldsymbol{y}$ - the total space being 12800 points. In order to predict across this design parameter space an emulator is trained on a five simulator evaluations from a generalised Latin hypercube design where for each evaluation a 512 point displacement history is generated.

$$
\boldsymbol{F}=m \ddot{\boldsymbol{y}}+c \dot{\boldsymbol{y}}+k \boldsymbol{y}+k_{3} \boldsymbol{y}^{3}
$$

The duffing oscillator in this case study has a mass of $m=25 \mathrm{~kg}$ and a damping ratio of $\zeta=$ 0.8 with which the damping coefficient $c$ is calculated. The forcing $F$ is a 512 point random-phase multisine input [21] displayed in Fig. 5. The grid of stiffness and cubic stiffness coefficients used to test the performance of the emulator are $k=\{600,650, \ldots, 800\} \mathrm{N} / \mathrm{m}$ and $k_{3}=\left\{4 \times 10^{5}, 5.25 \times\right.$ $\left.10^{5}, \ldots, 9 \times 10^{5}\right\} \mathrm{N} / \mathrm{m}$.

The sparse GP emulator predictions, when trained using 250 inducing points on the 5 point Latin hypercube design, produced predictions across the design parameter grid with a NMSE of 1.33 show- 


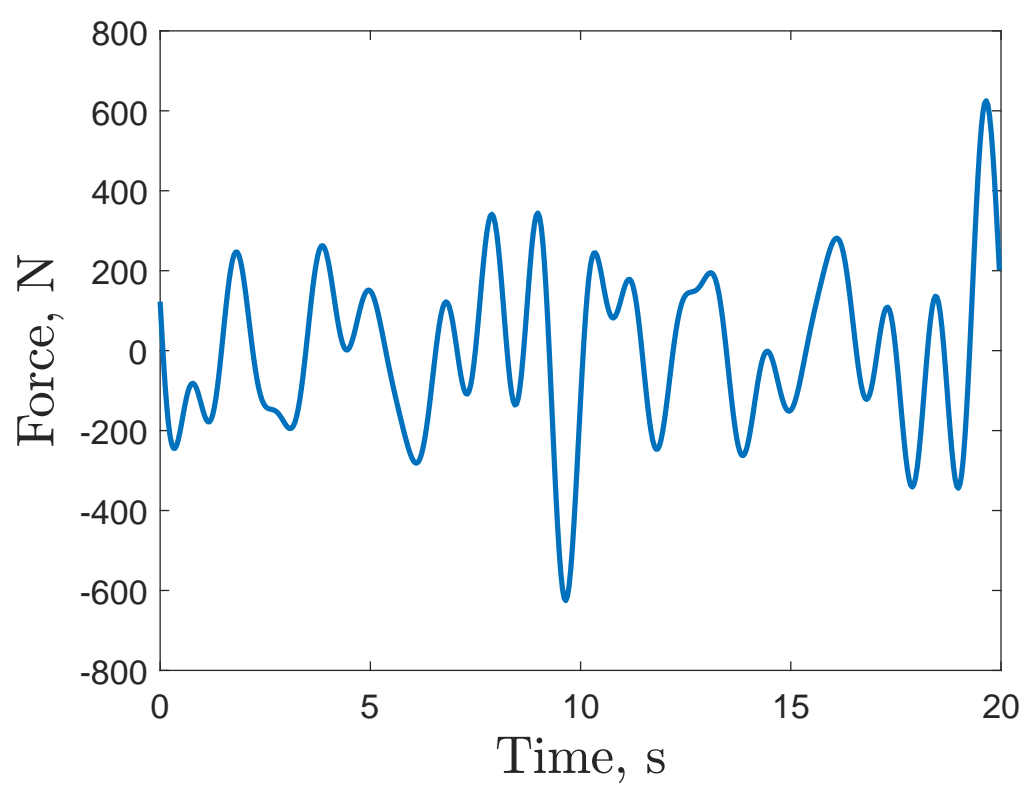

Fig. 5: Random-phase multisine force input for the duffing oscillator.

ing an very good fit to the data. Due to the large space being predicted a zoomed in example of the prediction is presented in Fig. 6. The results show that sparse GPs can be used to capture large design parameter spaces, and therefore could be implemented inside an uncertainty quantification or design optimisation technique. The example also shows that the code uncertainty predicted by the emulator reflects both the sparse assumption and the size of the training set. In an optimisation context, if incorporated this uncertainty would ensure that parameter space is not excluded due to poor emulation.

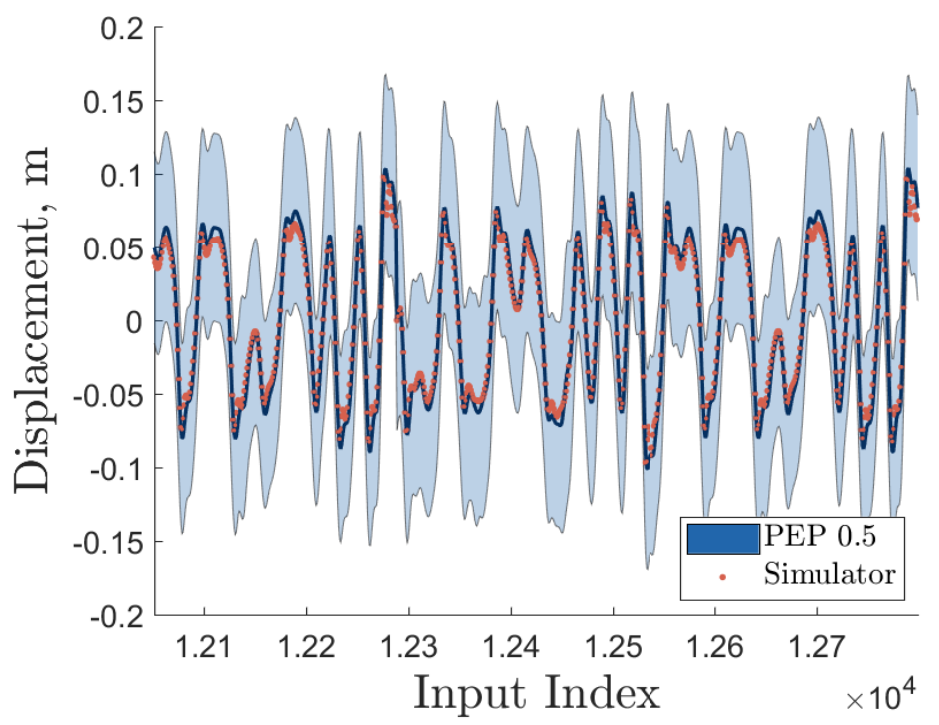

Fig. 6: A zoomed in section of the sparse GP emulator (PEP with $\alpha=0.5$ and 250 inducing points) for the duffing oscillator displacement output across the $5 \times 5$ design parameter grid of stiffness $k$ and cubic stiffness $k_{3}$ values. 


\section{Conclusions and Further Work}

GP emulators are powerful tools for exploring outputs from an expensive simulator. The approach can be especially useful in a Bayesian optimisation or Bayesian history matching settings. Here code uncertainty can be informative as to where to sequentially add the next simulator runs. The problem with GP models are that they are order $\mathcal{O}\left(N^{3}\right)$ to train and are order $\mathcal{O}(N)$ and $\mathcal{O}\left(N^{2}\right)$ for mean and variance predictions per test point. In settings where the simulator is very expensive and the design space is small a full GP solution is practical. However, some applications require a large number of simulator runs if the parameter space is high dimensional and necessitates a combinatorial approach to selecting inputs to understand the simulator output. In this scenario, sparse GP models are appropriate as they reduce the computational load of training to $\mathcal{O}\left(N M^{2}\right)$ where a prediction is of the order $\mathcal{O}(M)$ and $\mathcal{O}\left(M^{2}\right)$ for the mean and variance respectively. Sparse GPs are also useful when a large number of predictions are required, as is the case in Bayesian optimisation and Bayesian history matching, where a moderate computational saving can be made.

There are two main approaches to sparse GPs, model or posterior approximation approaches. It has been demonstrated that model approximation methods will result in overfitting, due to the inducing points being parameters of a parametric model. For this reason, both a DTC and FITC approach are often not appropriate for sparse GP emulators. On the other hand, posterior approximations provide a solution by treating the inducing points as part of the inference, keeping the model exact and the method non-parametric. The two main approaches to posterior approximation methods are VFE and PEP. It has been shown that VFE will often smooth through the data, due to the trace penalisation term, resulting in predictions that overestimate the noise. PEP resolves these issues by reducing the penalty and generally outperforming VFE (especially when $\alpha=0.5$ ). Therefore, it is recommended that when sparse GPs are appropriate a PEP approximation should be used for generating emulators as this provides satisfactory performance.

Further research should be conducted into creating local approximations whilst retaining a global predictive quality. This could be achieved with a partially independent training conditional (PITC) approach where inducing parameters are fixed at known training points where the simulator output is of interest - for example where a maxima or minima is located. This would provide benefits in using the computational savings of a sparse approach will producing full GP inference around local regions of interest.

\section{References}

[1] Jerome Sacks, William J. Welch, Toby J. Mitchell, and Henry P. Wynn. Design and Analysis of Computer Experiments. Statistical Science, 4(4):409-423, 1989.

[2] Anthony O’Hagan. Bayesian analysis of computer code outputs: A tutorial. Reliability Engineering \& System Safety, 91(10-11):1290-1300, oct 2006.

[3] Marc C. Kennedy and Anthony O'Hagan. Bayesian calibration of computer models. Journal of the Royal Statistical Society: Series B (Statistical Methodology), 63(3):425-464, 2001.

[4] Philipp Hennig and Christian J. Schuler. Entropy Search for Information-Efficient Global Optimization. Journal of Machine Learning Research, 13:1809-1837, dec 2012.

[5] Jasper Snoek, Hugo Larochelle, and Ryan P. Adams. Practical Bayesian Optimization of Machine Learning Algorithms. In NIPS, pages 2951-2959, jun 2012.

[6] Ioannis Andrianakis, Ian R. Vernon, Nicky McCreesh, Trevelyan J. McKinley, Jeremy E. Oakley, Rebecca N. Nsubuga, Michael Goldstein, and Richard G. White. Bayesian History Matching of Complex Infectious Disease Models Using Emulation: A Tutorial and a Case Study on HIV in Uganda. PLoS Computational Biology, 11(1), 2015. 
[7] Paul Gardner, Charles Lord, and Robert J. Barthorpe. Bayesian History Matching for Forward Model-Driven Structural Health Monitoring. In Proceedings of IMAC XXXVI, 2018.

[8] Anthony O’Hagan and John F. C. Kingman. Curve Fitting and Optimal Design for Prediction. Journal of the Royal Statistical Society: Series B (Statistical Methodology) (Statistics in Society), 40(1):1-42, 1978.

[9] Carl E. Rasmussen and Christopher K. I. Williams. Gaussian processes for machine learning., volume 14. 2004.

[10] Joaquin Quiñonero-Candela and Carl Edward Rasmussen. A Unifying View of Sparse Approximate Gaussian Process Regression. Journal of Machine Learning Research, 6:1939-1959, 2005.

[11] Edward Snelson and Zoubin Ghahramani. Local and global sparse Gaussian process approximations. In 11th International Conference on Artificial Intelligence and Statistics, 2007.

[12] Thang D. Bui, Josiah Yan, and Richard E. Turner. A Unifying Framework for Gaussian Process Pseudo-Point Approximations using Power Expectation Propagation. Journal of Machine Learning Research, 18(1):3649-3720, 2017.

[13] Edward Snelson and Zoubin Ghahramani. Sparse Gaussian Processes using Pseudo-inputs. In NIPS, pages 1257-1264, 2005.

[14] Michalis Titsias. Variational Learning of Inducing Variables in Sparse Gaussian Processes. In AISTATS, volume 5, pages 567-574, 2009.

[15] James Hensman, Nicolo Fusi, and Neil D. Lawrence. Gaussian Processes for Big Data. In Twenty-Ninth Conference on Uncertainty in Artificial Intelligence (UAI2013), pages 282-290, sep 2013.

[16] Hildo Bijl, Jan Willem van Wingerden, Thomas B. Schön, and Michel Verhaegen. Online sparse Gaussian process regression using FITC and PITC approximations. IFAC, 48(28):703-708, 2015.

[17] Noel Cressie. Statistics for Spatial Data. Terra Nova, 4(5):613-617, 1992.

[18] Ioannis Andrianakis and Peter G Challenor. The effect of the nugget on Gaussian process emulators of computer models. Computational Statistics \& Data Analysis, 56(12):4215-4228, dec 2012.

[19] Leonardo S. Bastos and Anthony O'Hagan. Diagnostics for Gaussian Process Emulators. Technometrics, 51(4):425-438, 2009.

[20] Matthias Bauer, Mark van der Wilk, and Carl Edward Rasmussen. Understanding Probabilistic Sparse Gaussian Process Approximations. In NIPS, pages 1533-1541, 2016.

[21] Rik Pintelon and Johan Schoukens. System Identification: A Frequency Domain Approach. 2012. 